\title{
interview
}

\section{Enhancing emission}

\author{
Andrei Faraon from Hewlett-Packard describes how he and his colleagues achieved an eightfold \\ enhancement to the zero-phonon emission from single nitrogen-vacancy centres in diamond.
}

\section{What is your work about?}

Nitrogen-vacancy (NV) centres are photoluminescent point defects in diamond that comprise a substitutional nitrogen atom next to a carbon vacancy. NVs are potentially interesting for quantum information and sensing applications because they can be individually optically addressed and have some of the longest electron spin coherence times among all solid-state quantum systems. The most promising schemes that use NVs for optical quantum information processing involve interfering photons emitted by the NVs themselves. However, such schemes emit only around 3\% of their photons at the zero-phonon line (ZPL), a small wavelength range that corresponds to direct transitions between excited states and ground states. The rest of the light is emitted in a broad spectrum known as the phonon sideband, which cannot be used for interference-based measurements. Our work demonstrates that emission at the ZPL from NVs in single-crystal diamond can be enhanced by coupling it to the mode of an optical microresonator fabricated directly in the diamond. This achievement will be essential for future quantum photonic devices based on NVs.

\section{How is your approach different} from others?

Researchers have enhanced emission at the ZPL from NVs in diamond nanoparticles by coupling the NVs to optical resonators. However, NVs in diamond nanoparticles generally have poor spectral properties, unlike their counterparts in single-crystal diamond. Although many groups have attempted to fabricate photonic devices in single-crystal diamond, these attempts were not successful enough to produce resonators with high quality factors. In our experiment, we fabricated the resonator directly inside single-crystal diamond. Our approach is unique in that the NVs are located inside the resonator where the optical field is intense, thus allowing good coupling efficiencies to be achieved. The emission couples to the well-defined mode of the optical resonator, which can then be coupled to an on-chip network.

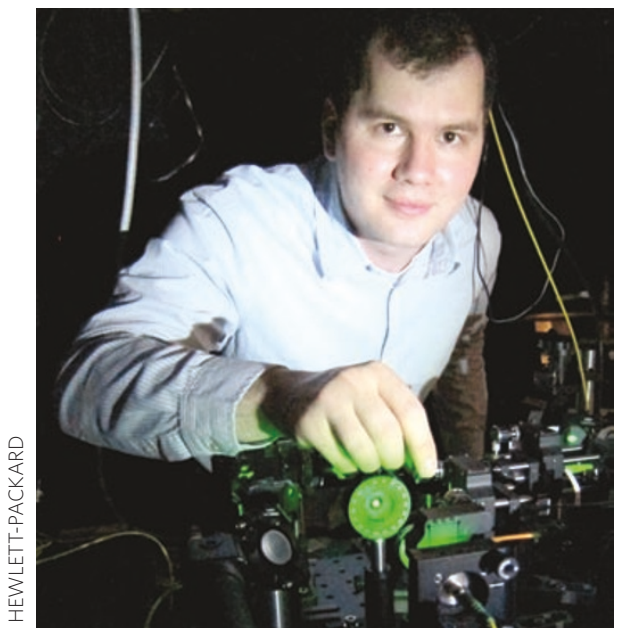

Andrei Faraon and colleagues exploit the region of intense electric field inside a resonator to enhance the emission from nitrogen-vacancy centres.

What is the physical mechanism behind the work?

The physical mechanism that causes the enhancement is known as the Purcell effect, which is manifested for emitters located in resonators with small optical mode volumes. A quantum emitter emits a single photon when decaying from an excited state to the ground state. If the emission occurs in a resonator that has a high quality factor and a small mode volume, the electric field that builds up during the emission is strong enough to enhance the spontaneous emission rate.

\section{What have you achieved?}

Coupling the emission to the resonator enhances the spontaneous emission rate in the ZPL while keeping the spontaneous emission rate in the phonon sideband constant. Thus, the number of photons emitted from the ZPL per unit time is enhanced. Using this technique, we were able to enhance the ZPL emission from 3\% to $25 \%$.

\section{Were there any difficulties?}

There were difficulties in both the fabrication and measurement of the devices. First, we had to develop a new technique for fabricating single-crystal diamond devices, which involved placing a 5 - $\mu \mathrm{m}$-thick diamond membrane on top of a silicon dioxide substrate. In terms of measurements, successfully coupling the ZPL to the resonator required the use of cryogenic temperatures to minimize the spectral width of the ZPL. We also had to use special spectral filtering and stabilization techniques to carry out the measurements.

\section{What are the potential applications of your findings?}

Our work demonstrates the potential for building sophisticated optical quantum networks in diamond. This will be essential for constructing quantum information devices and may also be helpful for developing efficient sensing devices based on NVs or other photoluminescent point defects in diamond. Although our work mainly focuses on a photonic platform for quantum information applications, we believe the approach can be extended beyond quantum information. For example, many interesting sensing devices based on photoluminescent point defects in diamond have been proposed but are limited by the amount of photons that can be collected. Our approach is also potentially scalable, which could dramatically advance the field of integrated diamond photonics.

\section{How can the technique be improved?} Our work is a proof-of-concept demonstration for fabricating quantum photonic devices in single-crystal diamond. However, there are several technological challenges that must be overcome before complex photonic networks can be built in this material. Diamond membranes with better thickness uniformity are needed, and techniques for site-specific implantation of single NVs must be developed. This will enable us to fabricate devices in which a single NV is deterministically coupled to a microresonator. Straightforward improvements in device quality could also be achieved by experimenting with the fabrication procedures.

\section{INTERVIEW BY RACHEL WON}

Andrei Faraon and co-workers have a Letter on zero-phonon emission from a diamond cavity on page 301 of this issue. 